\title{
Language as an Emergent Function: Some Radical Neurological and Evolutionary Implications
}

\author{
Terrence W. DEACON
}

BIBLID [0495-4548 (2005) 20: 54; pp. 269-286]

\begin{abstract}
Language is a spontaneously evolved emergent adaptation, not a formal computational system. Its structure does not derive from either innate or social instruction but rather self-organization and selection. Its quasi-universal features emerge from the interactions among semiotic constraints, neural processing limitations, and social transmission dynamics. The neurological processing of sentence structure is more analogous to embryonic differentiation than to algorithmic computation. The biological basis of this unprecedented adaptation is not located in some unique neurological structure nor the result of any single mutation, but is vested in the synergistic interaction of numerous coevolved neurological biases and social dynamics.
\end{abstract}

Key words: coevolution, emergence, self-organization, semiotics, differentiation, neural development

\section{Introduction: The fallacy of reverse-engineering}

There can be little doubt that the development of formal generative linguistic analysis has been the most important advance in the study of language in the last half century. These formal models predict the structure of linguistic output both accurately and compactly, including its open-ended generativity. The elegance and compactness of these formal theories are a function of good reverse-engineering. It would be odd if these theories exhibited poor predictive power, considering that they were consciously designed to yield results consistent with vast amounts of known (and self-generated) linguistic data. Thus it seemed reasonable to extrapolate from this model to the evolutionary and neurological origins of language. But after nearly a half-century of unsuccessful efforts to find the neurological and genetic counterparts to universal grammar or the algorithms comprising the principles and parameter-setting mechanisms of language it may be time to reflect on why neurobiology and formal linguistics have not converged.

Paradoxically, the successes of formal generative linguistic theories may have impeded progress toward understanding language neurology and language evolution, even while they have provided such remarkably sophisticated tools for the description of language structures. This is because the apparently remarkable adequacy of formal models to account for the complexities of language structure have contributed to an unwarranted assumption that language can be studied as though its structure was designed by a kind of instruction logic, as are other formal systems. Yet despite compelling evidence that language has a formal structure consistent with top-down rulegoverned systems, its status as an evolved — not designed - biological phenomenon raises serious questions about the plausibility of extrapolating from this descriptive analysis to a theory of language processing. 
This problem shares some ironic parallels with mid $20^{\text {th }}$ century debates about the immune response. The generativity and flexibility of the immune system was then thought to require some mechanism for instructing immune cell molecules to conform to the specific structure of disease antigens in order to be able to bind to them selectively. Immune potential could be encoded in the genome but the complexity of this code would need to be enormous. Because specific immunity can be acquired, antibody structure might also be "learned" by matching to antigen structure on exposure. The solution required abandonment of both instruction theories. Generation of adapted antibodies results from blind variation, serendipitous matching, and differential proliferation of the best fit immune molecules in response to antigen exposure. With respect to language, this is a cautionary tale about the (in this cases erroneous) assumption that only an instructional mechanism can accomplish this degree of functional precision and generativity.

Probably the most compelling reasons to avoid assuming a simple correspondence between linguistic models and neural processing logic, however, comes from comparative neuroanatomy. Despite the obvious human uniqueness of language, no unprecedented new brain structures distinguish human from nonhuman primate brains. Even the so-called language areas of the cerebral cortex (e.g. Broca's and Wernicke's areas) have been shown to derive from primate homologues sharing, positional, cytoarchitectyonic, and connectional patterns with their human counterparts, despite not subserving language or even vocal control (e.g. see Deacon, 1997; 2004). Although brains also have functional hierarchies that divide into interdependent functional systems, this reflects the constraints of embryology and phylogeny and not any linguistic functional role. So language processing is ultimately carried out with the same brain structures and functional logic that other primates use for nonlinguistic functions.

\section{Mechanical versus organic analogies}

The difficulty may, however, be more serious. There is reason to believe that these formal models predict generative architectures that substantially reverse the order of the critical generative processes.

Linguistic logic is based on rules for manipulating basic units (e.g. phonemes, morphemes, grammatical operators, etc.) to create larger complexes (e.g. phrases and sentences). Linguistics is thus largely an enterprise of top-down analysis. For linguists, full-fledged language phenomena are the starting points of analysis. The basic units that comprise patterns of use and with respect to which linguistic judgments are made are morphemes, grammatical operators, syntactic relationships, and so forth. Most linguistic theories assume the primacy of these linguistically defined language units, and differ primarily in their analysis of operations applied to them. This is equally true for algorithmic and constraint-based approaches to grammar, and to a lesser extent for cognitive grammars. An exemplar of a classic phrase structure analysis based on rewrite rules is depicted in Figure 1. This approach is characterized by instructions for generating phrases and sentences by combining lexical elements. It assumes a separation of syntactic from semantic operations, and these from phonological operations. 
As a result, there must also be a system of bridging rules for linking these three derivational systems. Some of the computational complexity of this approach is avoided by constraint-based approaches to grammar. A system of combinatorial instructions is unnecessary if one assumes that the basic lexical building blocks come with implicit structure - analogous to chemical valence - that limits their combinatorial possibilities (see Figure 2). Thus phrases and sentences can self-assemble without extrinsic instruction, driven only by elicitation of the lexical units and a generic "merging" operation. This shifts the burden of structural information to the lexical level, and is in accord with the psychological experience of unmonitored spontaneous sentence generation in which most of the conscious attention is directed toward content word choice. But it even more emphatically requires an explanation of the basis of these constraints (e.g. innate grammatical categories) and the mechanism by which possible merges are allowed or disallowed. All approaches that assume that sentences are neurologically built-up from more basic non-composite grammatical units, must provide a separate account of the source of these prior rules or constraints and must incorporate a mechanism for sentence construction or combinatorial constraint, respectively. Ultimately, we must map all to neural processing units.

The assumption that minimal lexical units are primary and linguistically composite structures are derived from combining these more basic units seems too obvious to question. Most sentences are novel productions, while all words and grammatical markers have ancient origins. How could sentences ever be prior to the words that compose them, either historically or mentally? This compositional analysis is consistent with a formal or engineering analogy for sentence generation. In such systems, a set of design instructions (or assembly constraints, or both) and a set of component parts precede all operations. Complex structures are accurately modeled by combining components according to these rules. So it seems natural to assume that the neural production of sentences should also proceed this way.

Now contrast this logic with the logic of evolution, embryonic development, or the development of brains (discussed below). Biological structure generally arises from bottom-up differentiation processes. This is the inverse of top-down instruction and the imposition of combinatorial order on component parts. In biological processes, functional parts (e.g. cells, organs, limbs) are not collected together and then assembled to make larger functional wholes. Undifferentiated wholes (e.g. a fertilized ovum, a gastrula, and embryo) precede their differentiation into composite structures, even though these also involve multiplication and subsequent differentiation of similar components from this beginning. The very few special exceptions (e.g. the eukaryotic cell) involve symbiotic relationships (e.g. mitochondria evolving from endosymbiosis) of already functionally complete wholes. In biology, functional integration is primitive, not imposed. Even if genes are construed as instructions, they are only instructions for molecular structure - providing biases and constraints on potential molecular interactions. To get from this to higher-order functional organization many levels of emergent cellular-molecular interactions must intercede. Thus in developed organisms earlier less differentiated stages (whether in phylogeny or embryology) are visible only 
as a sort of dim palimpsest, buried in and modified by many stages of subsequent differentiation. So although we often describe the structures and functions of bodies as though they are marvels of engineering, they achieve this appearance by means almost diametrically opposed to engineering design.

Noam Chomsky's (1957) early demonstration that syntactic manipulations of language could be modeled with considerable adequacy as rewrite operations (as could be implemented as a Turing machine) was a compelling indication that language is special, and it suggested an almost irresistible comparison with other formal systems of computation. Moreover, language is a social phenomenon that is potentially responsive to pressures for rule-governed standardization (of course this typically imposes one naturally evolved language regularity on the behavior of speakers with slightly different variants). But as compelling as this is, the fact remains that language is a spontaneously evolved phenomenon produced by a biological organ, and biology is not engineering.

\section{Brain development as self-organization and micro-evolution}

It has become increasingly clear that the vast majority of the 'design information' expressed as brain structure is not encoded in genes. Mouse brains, which are roughly the size of the last digit of your little finger, develop using roughly the same number of corresponding genes as does the human brain, which is roughly the size of a pineapple. With each neuron interconnected with roughly a thousand others in both kinds of brains, the information embodied in these two structures differs by many orders of magnitude. So where does the extra information come from to build human brains?

The answer does not come from reapportioning influences of nature versus nurture, but rather from neither. The extra structural information arises spontaneously, not by magic or divine intervention, but by a process that, like evolution, can spontaneously generate adaptive correspondences and novel complex structure and function without external guidance. Indeed, brain development resembles a kind of microevolution in many important respects. And both evolution and embryology take advantage of a variety of spontaneous ordering tendencies as well.

The way that open dynamical systems fall into orderly patterns without extrinsic imposition of these regularities has come to be called self-organization, and the dynamical structures that arise from such processes (as well as from evolutionary processes) are generally described as emergent (for an overview see Deacon, 2003b). Examples of autonomous pattern-generation can be seen in the formation of hexagonal convection cells in a heated liquid (Bénard cells), the individually idiosyncratic yet hexagonally symmetrical growth of snow crystals, and the interwoven spirals of seeds, leaves, and petals that spontaneously organize to exemplify the Fibonacci number series (which is not explicitly encoded in the plant's genes). These regularities of structure and function are not prefigured in any antecedent instructional process but come into existence dynamically as the repeated interactions of components gradually reinforce some structural biases and damp others. Self-organized regularization can lend itself to selection dynamics as different patterns of interaction are pitted against one another in a 
larger "ecology" that is more concordant with some compared to others. This interplay of self-organization and selection processes is responsible for much of the emergent structure and adaptive complexity of brains.

For example, in the process of brain development, patterns of connection are initially generated by the self-organizing interactions of axonal growth processes between regionally organized distinct cell populations (these in turn arose from self-organizing and competing cell proliferation and interaction processes). Converging axons then compete with one another in an 'ecology' of signal-processing demands. The resulting selection process culls many and preserves other cells and connections that are more synergistic in activity patterns. In this way, much like phylogenetic evolution, the developing nervous system can augment the biasing influences of the genes by using this as a base from which to 'explore' adaptive correspondences between different neuronal populations, between regionally different signaling patterns, and between organism and environment. This contextually sensitive sculpting of cell populations and connections results in the spontaneous emergence of complex functional synergies as the developing brain adapts to the body it finds itself in.

This should not be misunderstood to mean that brains of humans and brains of chimpanzees, for example, mostly differ with respect to these plastic connections. Human brains resemble other human brains, not chimp brains, because of the influence of genetic differences. Self-organization can in fact augment the subtle biases contributed by gene differences affecting cell-cell interactions to produce large-scale systemic change. Physical systems, like snow crystals for example, share remarkable family resemblances despite diverse conditions of origin, because of (not in spite of) the regularizing affect of self-organization. Though stochastic factors may make the fine details unpredictable, general patterns are reliably produced even though this emergent structure is not predetermined. Similarly, selection processes tend to produce convergence toward common forms (e.g. streamlining in diverse aquatic species) despite diverse origins and substrates. This is an important point, which is often misrepresented by overzealous critics of genetic determinism. Emergent structures are often highly predictable and can converge on universal features, even from quite different initial conditions. This is of course relevant to language regularities, as well.

When brain development is viewed through the prism of self-organization and selection processes, little remains of the engineering logic that is so familiar, and yet the result can still be precisely organized. Brain development demonstrates that just because a biological structure is highly predictable, complex, and systemic in organization we need not appeal to algorithmic or instruction logic to explain this fact. Moreover, the extensive role played by self-organizing dynamics in brain development should make us highly suspicious of engineering analogies used to explain brain functions like language production.

\section{The emergent architecture of neurolinguistic processes}

Languages and human language abilities have evolved spontaneously. Like other naturally evolved complex systems we should expect to find that they exhibit the hallmarks 
of this undirected process reflected in an emergent architecture. Language has an emergent architecture to the extent that its structure is a product of spontaneous bottom-up self-organizing interactions, not top-down imposition of structure or constraint by any pre-existing template. This requires conceiving of basic linguistic units as differentiated end-products of a cognitive process rather than as fundamental atoms of analysis.

To do this we need to analytically de-differentiate the many levels of a speech act, but this introduces a conceptual difficulty. We cannot start with the familiar components of expressed language, but must derive these from something far less concrete that has troubled psychology since its inception: the form of a thought before it is put into words. We find it difficult if not impossible to gain introspective insight into the nature of a word before it is formed, or the idea that a sentence conveys before it is encoded into words. Remarkably, for all the difficulty we have describing this, it is probably fair to say that a good deal, if not most, of our mental life is lived in this not quite articulated not quite formulated state.

This stage of cognition that serves as the nearly unconscious and automatic ground of language use is quickly and effortlessly resolved into words and sentences. We are usually entirely focused on aiming for and achieving expressive goals, not on selecting function words or following grammatical rules, and so long as these results are achieved without any serious hitch (e.g. because of content word-finding difficulties) the antecedent generative processes go unnoticed.

But if sentence structure is produced analogous to the way embryos develop, not as machines are built, then words and sentences must also begin as some less differentiated cognitive structures. Following this analogy we should consider language processing as though words and phrases differentiate from more inclusive generic precursors, which are the cognitive ancestors to structures with multiple component parts. These precursors are not, then, linguistic units, but rather more general cognitive, semiotic, and pragmatic structures. The apparently most elementary phonetic and morphological features of language are, in comparison, the most elaborated fractional distinctions to emerge from the progressive differentiation of these larger semioticneurological speech-act "embryos" (see Figures 3-5).

So we must begin with the most difficult step first: identifying these most abstract levels of what can be called the 'infralinguistic' hierarchy. The questions "What is a concept before it is expressed in words and phrases?" and "What is a proposition or request before it is phrased as a sentence?" must eventually be given serious attention as linguistic issues. The linguistic paradigm calling itself Systemic Functional Linguistics (e.g. Halliday, 1994) probably comes closest to this with respect to the analysis of communication processes in general, but parallels need to also be devised for the neurology of language processing (but see Brown, 1979 for a similar analysis).

Let's begin by considering a simple declarative sentence produced with the intention of describing some state of affairs. It may have a social context, which directs and constrains the kind of information that will best fit, such as being produced in response to a request or expectation. This will play a role in promoting one's change in 
arousal to speak and in eliciting certain memories or shifts of attention to relevant events. The social-pragmatic context includes a shift to a specific mode of communicating as well — e.g. providing or seeking information — and may consequently involve activating habits associated with this social role. This first stage is thus a social and pragmatic orientation stage that creates both a specific communicative frame and an arousal to act within it. Even merely interpreting someone else's speech requires establishing such a communicative framing of activity along with attention focused on certain expected content. It is what amounts to the act before it is initiated, the content before it is developed, the perception before it is sensed. It is a focused readiness and expectation with respect to social interaction.

Within this framing of social-communicative arousal what might be described as the "mood" of the speech- or interpretive-act is differentiated. This forms the minimally differentiated space of options from which further differentiation of content and expression can proceed. But the neural trace that constitutes this extrinsic framing does not get "passed on" to some subsequent brain structure for processing (as might be imagined for a component assembly process). Rather this "mood" needs to be maintained in parallel throughout the sentence generation processes and is updated only with a shift in communicative role. This requires a distinct neural substrate specialized for maintaining social orientation and arousal, and for monitoring conditions that would require a shift from this state.

Neurologically we should not expect to find that the areas of the brain associated with this phase of language behavior is specifically associated with speech production or comprehension per se. The arousal process almost certainly involves limbic structures and adjacent perilimbic cortical regions, as well as deeper brain structures associated with social arousal. Cortically this includes the anterior cingulate cortex, which mediates the arousal and monitoring process. These earliest phases are also comparatively slow-changing, so that that many sentences may be differentiated within a single generalized communicative mood. Later phases will correspond with progressively smaller units of a communicative act.

Damage affecting the brain regions associated with this arousal and monitoring of communication produces deficits that are seldom described in terms of aphasia, since word-choice, grammar, and phonetic decisions are unaffected. But more global disturbances of language are typical. Most notably, damage to midline frontal regions, including anterior cingulate cortex and supplementary motor area (especially bilaterally) is known to produce akinetic mutism. This has often been described as an inability to generate sufficient arousal-to-act because it can suddenly and transiently abate under high arousal conditions. In vivo imagery has shown that differentially elevated anterior cingulate activity is also associated with many language generation tasks, even when motor speech functions are controlled for (e.g. see Deacon, 1997). It is notable that midline frontal regions are also the only cerebral cortical regions from which primate vocalizations have been elicited by stimulation. Although speech cannot be elicited by cortical stimulation in humans (only blocked or modified), there have been reports of expletives being elicited by stimulation of limbic structures in human patients and 
such automatic arousal-correlated word production is often all that is spared in cases of severe global aphasia with massive damage to left perisylvian areas.

Within this mood frame the first specific orientation and expectations of the semiotic process are next generated. These include the intended goal of the communication and the selection of major categorical orientations relevant to this context. This is a phase of neural processing in which specific sensori-motor orientations relevant to the communication become more specified and need to be sustained in the face of competing and interfering alternatives.

These processes appear to involve sub-regions of prefrontal, parietal, and middle temporal cortex. These areas of cortex are mostly polymodal, judging from their primate homologues, and in classic (misleading) terminology were described as "association cortex." Consequently, damage to parietal and temporal areas tends to result in difficulties of maintaining attentional focus and distinguishing distinct objects of interest, respectively, especially when multiple modalities of discrimination are involved. Damage to lateral prefronal areas disturbs the ability to manage multiple competing sensori-motor attentional options, irrespective of salience, recency, or immediate reward contingencies. Prefrontal functions are sometimes referred to as 'working memory' because they involve the ability to hold many simultaneous competing predispositions in mind at once so that they can be assessed with respect to one another. In vivo imagery studies consistently show differential activation of the left ventral prefrontal region (anterior to what is more commonly defined as Broca's area) during wordassociation and memory tasks that involve rapid symbolically mediated decisions (see examples in Deacon, 1997).

With respect to language differentiation processes I think this can be understood as the phase in which predication differentiates out of a more general mood of communicative arousal. In many ways, the structure of predication has much in common with specific sensori-motor orientation. The logical structure of predication, formally symbolized by a function $F$ operating on one or more variables, in the form $F(x)$, or $F(x, y)$, etc., can be interpreted in semiotic terms as a symbolic relationship $F$ indexed to (pointing to) some locus or loci $(x, y)$ in the world, which may include loci within the physical communication process. In computational terms these comprise an operation and one or more 'slots' for pointing to the 'addresses' for the data to be operated on. In terms of semantic theory, $F$ is a predicate (e.g. verb) and $x$ and $y$ are arguments to which it applies (e.g. subject and object). In neurology we may see an analogy to the trace of a sensory or behavioral association and an attentional orientation toward some locus or loci to which it currently applies (ether external or internal to the organism).

In an essay concerning some semiotic constraints on language structure (Deacon, 2003c) I have argued that, because symbolic reference is "reference mediated by a system of symbol-symbol relationships," each symbolic function requires an associated indexical operation (described as a bound index) to point outside this system to objects of reference. Thus the utterance of a lone noun or verb almost never constitutes a completed act of communication. In other words it predicates nothing. It does not re- 
fer outside its implicit web-locus of symbol-symbol relationships. It is a fragment, lacking a necessary functional component that if present could constitute a sentence, proposition, request, order, and so on. But notice that when coupled with a pointing finger or uttered in a context where a specific object or state of affairs is obviously salient to the message recipient, this missing role is filled. The missing piece was thus playing an indexical role. This suggests that a complete symbolic act, so to speak, consists of a least one grammatical unit playing a symbolic role and another playing an indexical role. In terms of the $F(x, y)$ formalism, $F$ is the symbolic operation and $x$ and $y$ are serving as it bound indices. Even though these variables can be filled with other symbolic operations, these too must be individually indexed or reference fails.

To the extent that prefrontal, parietal, and middle temporal systems play critical roles in maintaining and deciding among specific orientation and action options, they are also relevant to the parallel operations on the virtual objects of symbolic reference as well. So, for example, the traces of object-attribute associations - and thus also the correlates for symbolic operations - are likely generated in posterior (temporalparietal-occipital and polymodal cortex), while the traces of orientational dispositions with respect to them - and thus also the neural correlates of indexical operations are likely generated and maintained in prefrontal cortical areas.

A classic descriptor of a global deficit common to patients with damage to one or more of these cortical systems is the 'loss of abstract attitude.' Though difficult to characterize, it generally refers to the overly literal way these patients approach language and behavior in general. This can be understood as an impairment of the ability to inhibit prepotent orientation to concrete sensori-motor associations compared to those that are more indirect and symbolically mediated. In classic aphasia terminology the so-called transcortical aphasias offer relevant correlates of damage to these still early stages of language differentiation. These leave perception, repetition, and production of speech intact but diminish control of these higher order content orientations. Posteriorly, this can produce confusions of associative analysis (transcortical sensory aphasia, and semantic aphasia) in which spurious interpretive substitutions may arise despite minimal nonsense paraphasia (nonword substitutions). For example, there may be word substitutions that reflect wildly divergent and incompatible categories, though they represent real words of the language and may even be appropriately inflected. Anteriorly, this can invove a weakened control of speech by predication with respect to merely reactionary or echolalic speech (transcortical motor aphasia) (for a classic review of aphasiology, discussed here and below, see Lecours et al., 1983).

Historically, these aphasic syndromes have come under critical scrutiny and have been considered by some to be of questionable validity as discrete syndromes or specific language disorders. This ambiguity reflects the semiotically more general character of this early phase of processing before word choice, syntax, and phonological realization are relevant. In this sense these kinds of deficits are often described in terms of ideational difficulties rather than linguistic impairments. Persistent transcortical motor aphasia also appears to require damage to underlying white matter and basal gan- 
glia structures, but as we will see, this is a feature that appears common to all frontal language deficits. This is often considered an argument against identifying frontal cortical structures with language processing per se, but this is an over reaction. The relatively greater importance of deep brain structures to frontal systems almost certainly reflects the far greater elaboration of cortical-basal-ganglia-thalamic-cortical circuits in frontal as compared to posterior cortex, and not a reduced importance of anterior cortical areas to language processing.

This predication-orientation phase of differentiation establishes the frame in which the first distinctively linguistic differentiation functions emerge, and for which there is general agreement that correlated impairments constitute true aphasias. These involve superior temporal regions of Wernicke's territory and the ventral frontal and prefrontal regions of Broca's territory (I prefer the designation 'territory' to the more common 'area' to avoid the implication of anatomical and functional homogeneity). In this phase of language differentiation the distinctions between frontal and posterior functions become more divergent and their functional interdependence decreases. This is in part a function of the decreased time domain for these functions to be performed, which limits the possibilities for complex interactions. Functional integration and coordination have however already been established by earlier phases that are more globally coupled.

The temporal regions adjacent to Heschl's gyrus (the site of the primary cortical auditory map) that comprise Wernicke's territory are extensively interconnected with middle temporal and inferior parietal polymodal areas. The predication-orientation established in these polymodal systems (which also activates parallel differentiation of more specific sensory imagery in modality specific areas to which they are also connected) superimposes corresponding constraints and biases on these more auditory specialized regions. This facilitates the activation of relevant classes of phonological traces for words (in anticipation of producing them or of the high probability of hearing them). Many cycles of word elicitation may occur within the frame of a single predication-orientation. This frame must also impose agreement constraints on the subsequent elicitation of words. Damage to cortical areas at this level of processing effectively interrupts this constraining and facilitation of word-sound expectation by predication relationships. Thus Wernicke's aphasics typically are not confused about their intentions to communicate and may understand much of the intention of others trying to communicate with them, but they tend to make both production and comprehension errors at or just below the word-formation level. While content words (carrying much of the load of predication) are inaccurately differentiated in these patients, resulting in frequent nonsense words and word substitutions that are in category but wrong in detail (e.g. 'chair' for 'bed'), function words ('this' 'is' 'it') tend to be retained and used fluently in appropriately structured phrase and sentence frames despite the paraphasias. We can describe this as a failure to differentiate the primary symbolic tokens despite maintenance of both the predicate frame and the indexical supports. 
Before analyzing these temporal functions further, consider the complementary role of frontal cortical systems associated with Broca's territory. The functions of this layer of processing take place within the predication-orientation frame established by prefrontal and polymodal posterior systems. The prefrontal contribution (as distinct from the parietal-temporal contribution) to establishing this predication frame is primarily with regard to the orientation or indexical component of this frame; i.e. the orienting with respect to different conditions and objects of attention. In general this aspect of the predication frame can be described as a schema for conditionally shifting orientation and redirecting attention. This is a fundamental feature of most complex learned behaviors in general. The subsequent phase of processing, characteristic of the cortical regions associated with Broca's territory, involve the differentiation of the elicitation and sequencing schemas regulating word production. This is accomplished by using the orienting constraints of the predicate frame to regulate predispositions activated by phonological cues from posterior processes and high frequency wordassociation habits keyed by prior word production. But this is one stage less differentiated than the motor production of the content words themselves, and can probably best be envisioned as generating the ordered slots into which these words will be inserted. But the indexical orientations of the previous level of differentiate into at least one form of overt motor output at this level: the production of function words, pronouns, and articles that serve as the markers for these syntactic slots and phrase transitions. These syntactic markers are the overt trace of an orienting and pointing schema that has differentiated in parallel with the posterior differentiation of word phonology.

These classic language areas thus represent the first level in which there is differentiation of linguistic units per se, but this interface should not be construed to be discontinuous from earlier stages. The various "component" linguistic units derive their referential power and combinatorial constraints from these many levels of infralinguistic processes. These lexical units may appear to self-organize as though they possessed intrinsic structure, but this apparent structure is rather a reflection of the fact that they are merely surface markers for the end stage of a cognitive differentiation process. Well before these specific lexical units are crystallized into aural-vocal traces, their combinatorial options have been prefigured by prior differentiation processes involving larger less differentiated semiotic units.

Broca's aphasia is typically identified with labored production of words and nonfluent speech, despite relatively spared vocabulary and comprehension. The nonfluency is also in part due to the absence of function words and grammatical markers. Although difficult production may indicate adjacent motor area damage, it may also simply be the result of the absence of sufficient cuing by syntactic markers whose function is to mark and point to the space where symbolic operations are required.

The subsequent, most differentiated levels of language processing — phonological analysis and vocal articulation of sentences - constitute a surface map on which linguistic tokens mark transition points of these deeply rooted continuous processes. The features that are articulated provide just enough cues of this hidden differentiation process as to allow others to independently recapitulate the process in parallel. 
Their efficacy is entirely dependent on a rich matrix of assumed interpretive commonality and anticipatory processing that will spontaneously differentiate with only minimal priming.

In summary, language is a neurologically emergent function because its structure is a product of the complex synergy that develops between these multiple systems and through levels of progressive neural differentiation. Each stage of differentiation involves correlated processing in corresponding levels of anterior (intention-action) and posterior (attention-sensory) cortical systems. Each establishes constraints and biases that are the ground from which the succeeding stage of differentiation begins. Subsequent phases involve both more specific and more rapidly differentiating and shifting processes, so that often a number of higher-order developments will occur under the relatively more persistent state of differentiation of the prior phase. This produces a multilevel nesting of more specific phases of differentiation within less differentiated frames, embedding shorter within longer time domains of operation. The processing of the exceedingly rapid phonological transitions of speech perception and the production of the rapid and subtle motor transitions of speech articulation are thus highly constrained by this nesting in a way that minimizes both the diversity of alternatives that must be anticipated with the demands of maintaining large scale functional integration.

\section{Implications}

So how does this translate into linguistic terms? Most current linguistic paradigms analyze grammar and syntax at one level — the level of differentiated linguistic structures - and derive rules and principles for handling all possible relations among these units. The consequence is that complex instructional architecture and massive processing capacity must be postulated to handle all the presumptive operations. This has further fueled the expectation that a language "processor" must be present in the brain and that only special language mutations of the genome could have made this possible. From a neural differentiation perspective, however, language processing develops through nested levels of operations on linguistic units of different orders of differentiation. The early stage levels are not explicitly represented as distinct linguistic units and largely involve neural systems that are strongly homologous in function to their nonhuman primate counterparts. Only the very last levels of functional differentiation correspond to linguistic compositional features, but this composite structure is a post hoc re-presentation of the entire differentiation hierarchy, not a recapitulation of it. The kind of question we must ask of our linguistic theory, then, is something like "What kind of semiotic unit — not linguistic unit — is a sentence or phrase?" This is a question of referential function and communicative pragmatics more generally. It suggest that a more sophisticated embedding of linguistic theory in semiotic theory is necessary in order for progress to be made in bridging the gap between linguistics and neurology.

The inversion of logic distinguishing generative formal analysis from biological differentiation logic also infects the study of language origins. The two paradigms of lan- 
guage generation correlate with two very divergent approaches to the evolution of its neural substrate. Did evolution produce an innate language faculty, in the form of a grammatical template or a set of grammatical categories with intrinsic combinatorial constraints (e.g. Pinker, 1994), or did it produce a synergistic combination of tweaks to the learning, mnemonic, automatization, and attentional processes of the human brain that together merely constrain and facilitate the emergence of language regularities (e.g. Deacon 1997, 2003a)?

A synergistic evolutionary account is at odds with many currently popular scenarios that suggest that language appeared rather recently and suddenly (50,000 to 100,000 years ago) as a result of one or a few critical mutations. Such "big bang" scenarios are only conceivable to the extent that the language faculty is treated as a monolithic instructional mechanism. But language is thoroughly integrated with other cognitive functions; it is developmentally canalized and robust in the face of early brain damage and genetic diversity; it is supported by many parallel supportive modifications in multiple cognitive systems; it takes advantages of unprecedented changes in the vocal system; and it exhibits a functional complexity that is unmatched by any other adaptation in ourselves or in other species. These well-known facts offer overwhelming evidence that the so-called 'language faculty' is a multifaceted system of adaptations that could only have been collectively shaped by selection with respect to language and each other over an extensive period of hominine evolution (Deacon 1997, 2003a).

Unfortunately, the study of language is not made easier by shifting to an emergence paradigm - indeed, it begs new questions, demands new methodologies, and requires a far more interdisciplinary approach than before. Moreover, this is not yet a theory, but merely a first speculative suggestion of another way of tackling this mystery. It is lacking in the linguistic details and in the descriptive power that is offered by even the simplest formal generative approaches. But the comparison is not fair. Formal theories have a post hoc tautological character - reverse-engineered by millions of person-hours from linguistic data. Their derivational logic is thus inevitably more elegant, their predictions are more accurate, and their domain of application is more comprehensive, so long as neural processing and evolvability considerations are not at issue. But ultimately these 'organic' considerations must trump predictive adequacy and formal elegance.

The formal design metaphor has diverted scientific attention and research resources from the implications of these biological considerations for a half a century, but refocusing attention on these factors does not mean turning our backs on formal linguistic, only abandoning the search for its literal counterparts in genes and the neural processes that generate language communication. I imagine that future linguists will look back upon this period in the history of the science and wonder why we didn't see the obvious utility in reserving engineering logic for the study of machines, formal logic for the study of computation, and organic logic for the study of brains and language. 

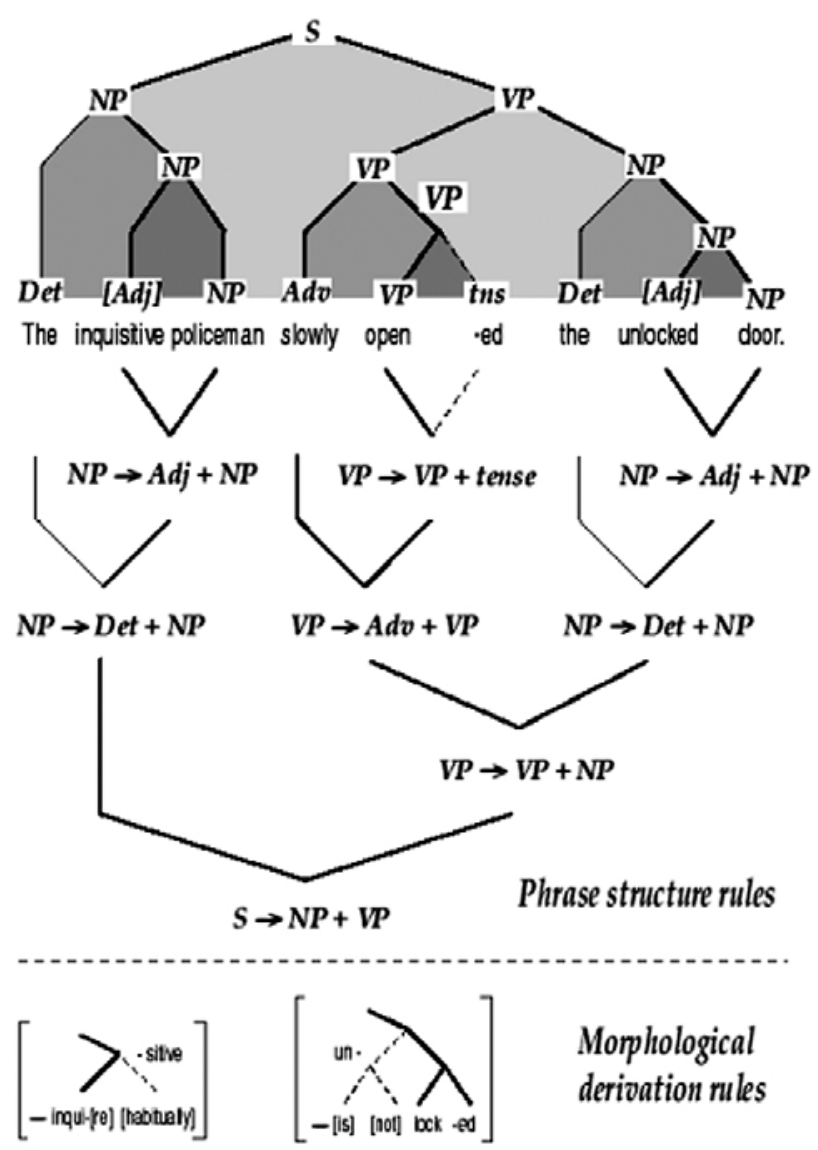

FIGURE 1. Algorithmic hierarchic "re-write" conception of sentence generation. Rules of composition and analysis are imposed top-down, allowing complete arbitrarity of the characteristics of lexical units. Lexical units still need to be identified with respect to grammatical categories, and then separate derivation of reference and phonology. Such an architecture is analogous to a Turing machine and if completely described can be modeled as a determinate machine as well as a formal system. 

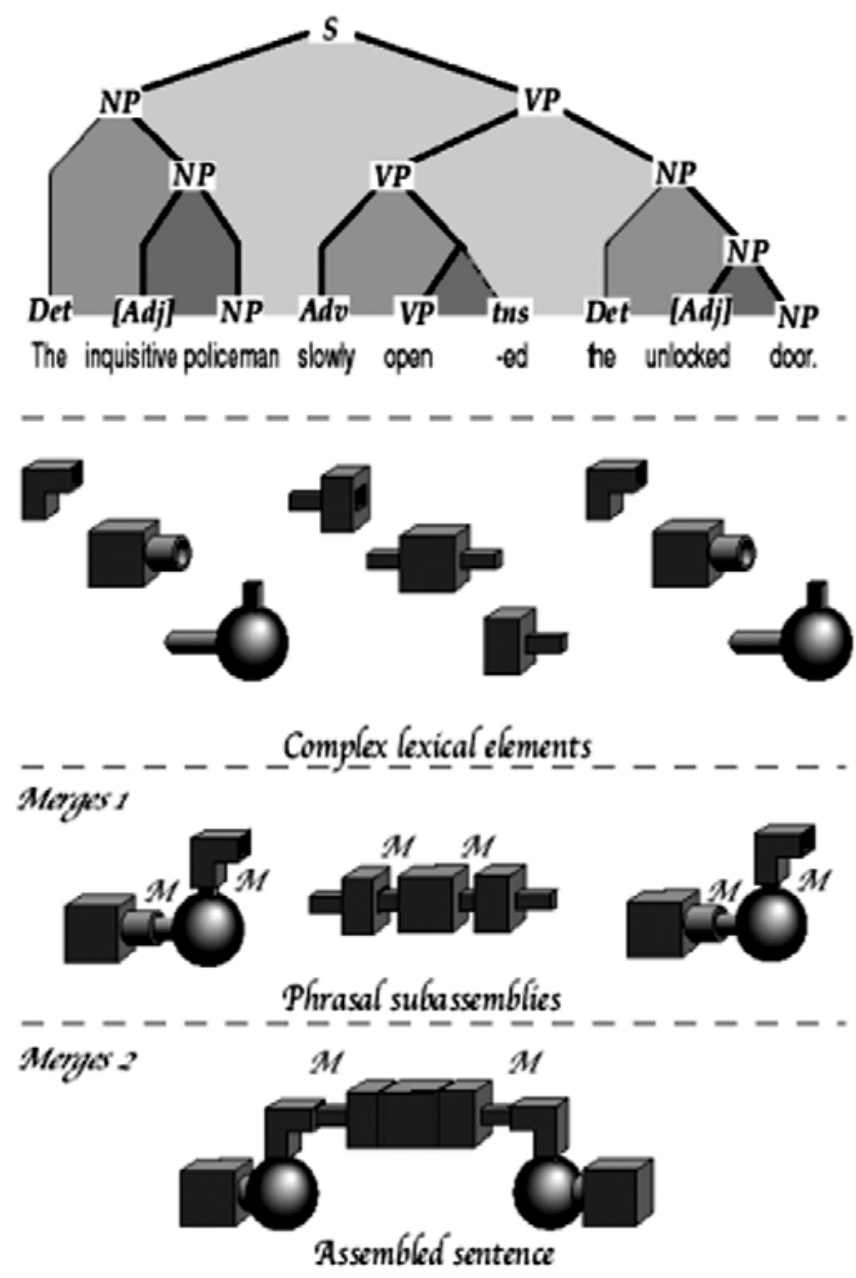

FIGURE 2. Constraint-based conception of sentence generation. By assuming that lexical units have constrained binding possibilities (on the analogy of specific types of atomic valence) phrasal assembly and sentential architecture restrictions can be derived spontaneously without top-down instructions (though order of operations may still require extrinsic constraint). However, this requires explaining how lexical items inherit this valence structure and how this is implemented other than by rules. 
[The[inquistive[policeman]] [[slowly[open][ed]]] [the[unlocked[door]]]

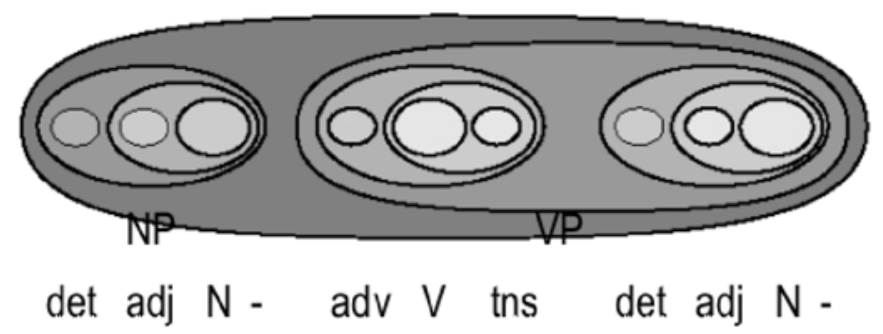

FIGURE 3. Hierarchic compositional analysis of sentence structure modeled by a Venn diagram (isomorphic with tree diagrams).

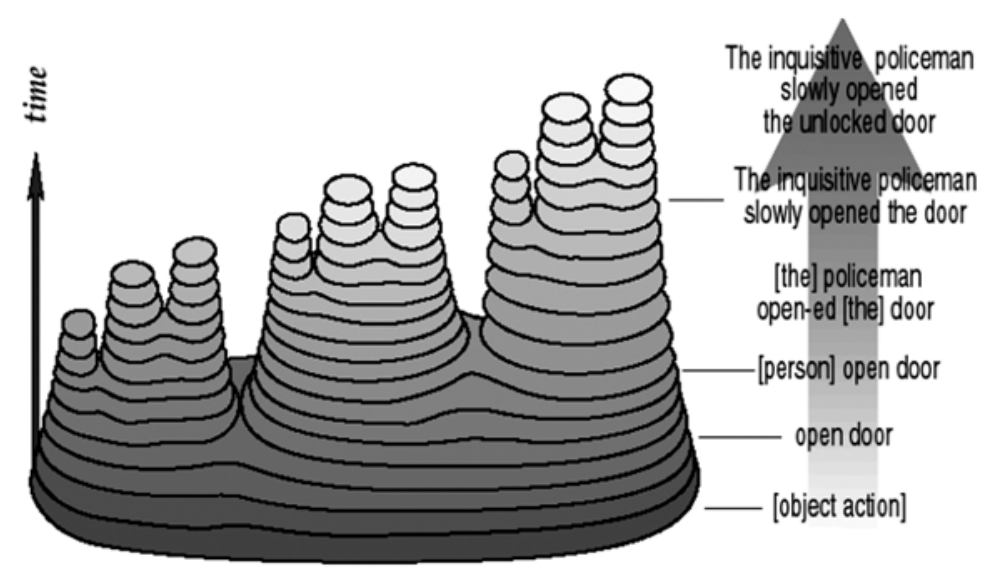

Articulatory production-delay-adjusted differentiation Sentence differentiation hierarchy

FIGURE 4. Expanding the embedding logic of the Venn diagram in a third dimension to represent a possible differentiation hierarchy helps in visualizing infralinguistic units of processing that may have their own derivation logic at that level of processing. These levels of differentiation can be glossed (right) as progressively differentiated sentences or holophrases. 

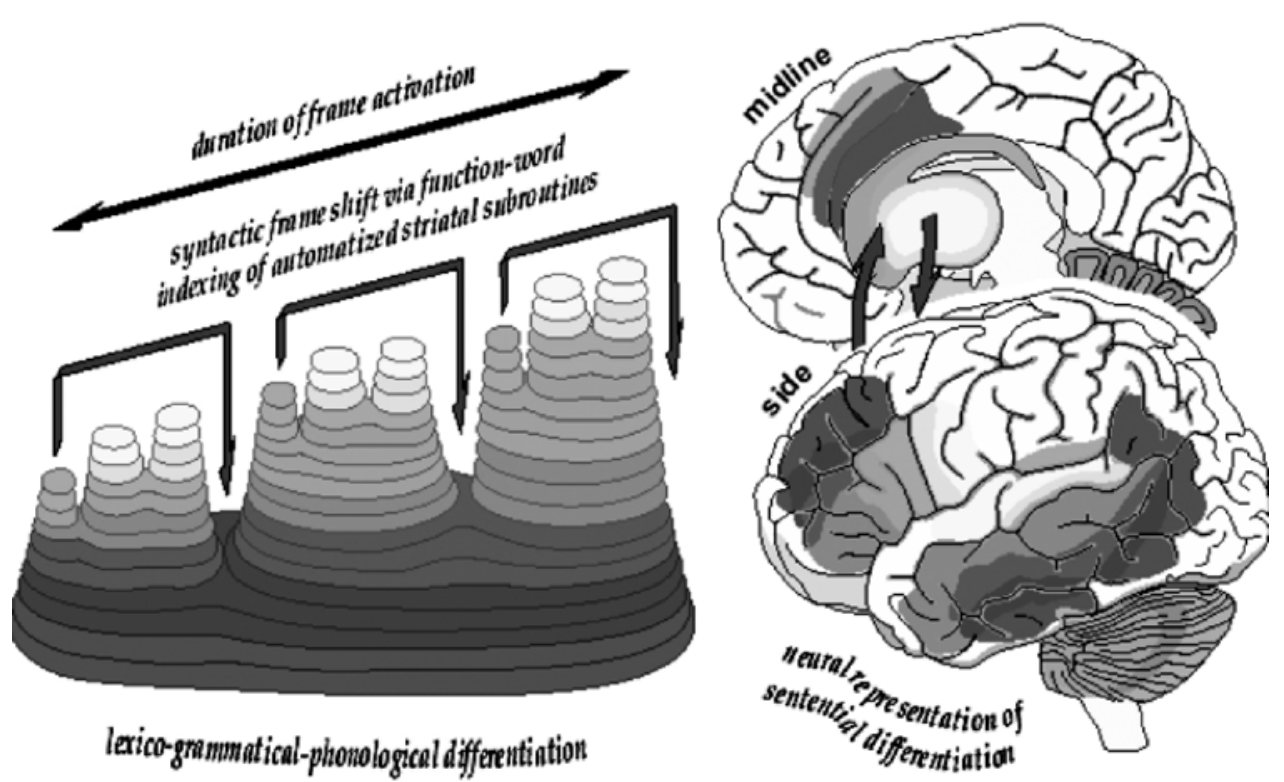

FIGURE 5. Mapping a differentiation hierarchy onto cortical areas (and some subcortical structures) that likely correspond to each given level of sentential differentiation. Note that differentiation converges toward classic language areas and ultimately terminates in auditory and motor areas. Each level of processing is complete in itself and does not produce or pass on a partially constructed sentence to the next. Rather each nested level is supported by level-specific habits acquired by language experience and these tendencies are differentially catalyzed or constrained by the state of the preceding level of differentiation and also by the biases introduced by the just preceding lower level process. Higher-order (less differentiated) processes also operate on a time scale that is slower and encompasses many lower level operations. These time domain differences may also express in language as short-term memory limitations that differ for different classes of language operations.

\section{REFERENCES}

Brown, Jason W. (1979). "Language Representation in the Brain," in H. Steklis and M. Raliegh (eds.), Neurobiology of Social Communication among Primates. New York: Academic Press, pp. 133-195.

Chomsky, Noam (1957). Syntactic Structures. Cambridge MA: MIT Press.

Deacon, Terrence (1997). The Symbolic Species: The Coevolution of Language and the Brain. New York: W.W. Norton \& Co.

- (2003a). "Multilevel selection in a complex adaptive system: the problem of language origins," in B. Weber and D. Depew (eds.), Evolution and Learning: The Baldwin Effect Reconsidered. Cambridge, MA: MIT Press, pp. 81-106.

- (2003b). "The hierarchic logic of emergence: untangling the interdependence of evolution and self-organization," in B. Weber and D. Depew (eds.), Evolution and Learning: The Baldwin Effect Reconsidered. Cambridge, MA: MIT Press, pp. 273-308.

- (2003c). "Universal grammar and semiotic constraints," in M. Christiansen and S. Kirby (eds.), Language Evolution. Oxford University Press. 
Deacon, Terrence (2004). "Monkey homologues of language areas: computing the ambiguities," Trends in Cognitive Science 8, 288-9.

Halliday, Michael A.K. (1994). An Introduction to Functional Grammar, 2nd ed. London: Arnold.

Lecours, André-Roch, F. Lhermitte and B. Bryans (1983). Aphasiology. London: Baillière Tindall.

Pinker, Stephen (1994). The Language Instinct: How the Mind Creates Language. New York: William Morrow.

Terrence DeAcon is Professor of Anthropology and Neuroscience at The University of California, Berkeley. His Ph.D. work at Harvard University traced neural connections in monkey brains that have language homologues. He has also been Professor of Anthropology at Harvard and Boston Universities, and a Researcher at Harvard Medical School exploring neural development and evolution using crossspecies neural transplantation. His 1997 book The Symbolic Species extrapolates from his neurological research to the origins of language.

ADDRESS: Department of Anthropology, Kroeber 329, University of California, Berkeley. Berkeley, CA 94720 USA. E-mail: deacon@berkeley.edu. 